Published in final edited form as:

Acta Obstet Gynecol Scand. 2008 ; 87(9): 979-981. doi:10.1080/00016340802334385.

\title{
Maternal Eating Disorders Influence Sex Ratio at Birth
}

\author{
Cynthia M Bulik, Ph.D. ${ }^{1,2}$, Ann Von Holle, M.S. ${ }^{1}$, Kelly Gendall, M.D., Ph.D. ${ }^{3}$, Kari Kveim Lie, \\ M.D. ${ }^{4}$, Elizabeth Hoffman, B.A. ${ }^{2}$, Xiaofei Mo, M.D. ${ }^{2}$, Leila Torgersen, Ph.D. ${ }^{5}$, and Ted \\ Reichborn-Kjennerud, M.D. 5,6,7
}

1 Department of Psychiatry, University of North Carolina at Chapel Hill

2Department of Nutrition, University of North Carolina at Chapel Hill

3Department of Internal Medicine and Women's Health, Virginia Commonwealth University Medical Center

4Division of Epidemiology, Norwegian Institute of Public Health

5Division of Mental Health, Norwegian Institute of Public Health

6Department of Psychiatry, University of Oslo, Norway

7Columbia University, Department of Epidemiology, New York, New York

\section{Abstract}

We explored sex ratio at birth, defined as the proportion of male live births, in women with anorexia nervosa, bulimia nervosa, binge eating disorder, and eating disorders not otherwise specified-purging type (EDNOS-P) relative to a referent group in a large population based sample of 38,340 pregnant women in Norway. Poisson regressions were adjusted for mother's age, pre-pregnancy BMI, lifetime smoking status, maternal education, income, marital status, gestational age, and parity. Lower proportions of male live births were observed in the anorexia and bulimia groups, while binge eating disorder and EDNOS-P were associated with a higher proportion of male births. These data suggest that maternal eating disorders may influence offspring sex and that the direction of effect may vary by eating disorder subtype. If confirmed, this finding could provide evidence in formulating hypotheses regarding the consequences of eating disorders and determinants of sex ratio at birth.

\section{Keywords}

anorexia nervosa; bulimia nervosa; binge eating disorder; sex ratio at birth

\section{Introduction}

Sex ratio at birth, also known as the secondary sex ratio, has been shown to be influenced by factors that are known to be disturbed in women with eating disorders including maternal diet, stress, maternal pre-pregnancy weight, body fat distribution, and hormonal factors (1-5). Particularly noteworthy, low maternal weight, a diet low in certain fats, higher stress levels, and lower androgen levels have been associated with fewer male births (1-5). As part of a larger investigation of the impact of eating disorders on pregnancy outcome in a large populationbased sample in Norway (6), we explored the impact of eating disorders on sex ratio at birth. To our knowledge, no reported association between maternal eating disorders and sex ratio at

Correspondence to: Dr Bulik, Department of Psychiatry, University of North Carolina at Chapel Hill, 101 Manning Drive, CB \#7160, Chapel Hill, NC 27599-7160, Voice: (919) 8431689 Fax: (919) 966-5628, e-mail: cbulik@ med.unc.edu. 
birth exists. Given known relations among diet, stress, and anorexia nervosa, we hypothesized that there would be a lower proportion of male births in women with anorexia nervosa.

\section{Material and Methods}

The data collection was conducted as part of the Norwegian Mother and Child Cohort Study (MoBa) at the Norwegian Institute of Public Health (7). The study has been approved by the Institutional Review Board of the University of North Carolina at Chapel Hill and appropriate regional committees for ethics in medical research and the Norwegian National Data Inspectorate.

MoBa is a prospective pregnancy cohort study. Pregnant women are recruited through a postal invitation after registering for a routine prenatal ultrasound at about 18 weeks' gestation. Participating women sign informed consent, donate blood and urine samples, and receive a questionnaire. The present study is based on the first questionnaire. The MoBa cohort is linked to Norwegian health registries, particularly the Medical Birth Registry of Norway (8) to capture pregnancy outcome variables.

Questionnaire 1 included items on eating disorders and behaviors derived from studies of the Norwegian Institute of Public Health Twin Panel (9) that reflected DSM-IV criteria for eating disorders (10). Diagnostic algorithms captured: broadly defined anorexia nervosa (DSM IV criteria excluding amenorrhea and endorsing a body mass index (BMI) $<18.5 \mathrm{~kg} / \mathrm{m}^{2}$ at the time of low weight); broadly defined bulimia nervosa (DSM IV criteria endorsing at least weekly frequency of binge eating and purging and categorized as any type, purging type, non-purging type); broadly defined binge eating disorder (DSM IV criteria with at least weekly frequency of binge eating in the absence of compensatory behaviors), and eating disorders not otherwise specified-purging type (EDNOS-P; purging at least weekly in the absence of binge eating). Anorexia nervosa was assessed before pregnancy only due to the practical difficulties in determining low weight in the presence of pregnancy-related weight gain. Bulimia nervosa, binge eating disorder, and EDNOS-P were assessed for both 6 months prior to pregnancy (retrospective assessment) and at the time of survey completion. Respondents were asked to distinguish between pregnancy-related nausea and vomiting and self-induced vomiting as a purging method. Self-reported weight and height were used to calculate BMI measures. A total of 38,340 respondents completed questionnaire 1 at a median of 18.6 weeks gestation (interquartile range 17.1-20.4 weeks and range 6.0-42.3 weeks).

The sex ratio at birth (the proportion of male live births) estimates were based on all women with singleton live births and eating disorder diagnoses. Poisson regression was used to estimate unadjusted and adjusted proportions and ratios with generalized estimating equations to estimate variance for this sample as described by Bulik et al. (6). Analyses were adjusted for factors known to affect sex ratio at birth including mother's age, pre-pregnancy BMI, lifetime smoking status, maternal education, combined income index, marital status, gestational age, and parity. All analyses were performed using SAS/STAT software for Windows and AIX, version 9.1 (11).

\section{Results}

Sex ratio at birth estimates are presented in Table 1. Unadjusted estimates showed 10\% and 9\% lower proportions of male live births for mothers with anorexia nervosa and bulimia nervosa, respectively, whereas maternal binge eating disorder and EDNOS-P were associated with $4 \%$ and $28 \%$ higher proportion of male births relative to the referent. After adjusting for relevant covariates, the risk ratio effects changed less than $2 \%$ in value for all eating disorder subtypes. 


\section{Discussion}

These findings suggest an association between eating disorders and sex ratio at birth in the hypothesized direction for anorexia nervosa and bulimia nervosa and in the opposite direction for binge eating disorder and EDNOS-P. The sex ratio at birth for the non-eating disorder population in these estimates, 1.04, closely matches previous Norwegian estimates of 1.05 (12) and 1.06 (13).

Hormonal and body composition changes, which are reported to affect sex ratio at birth as noted above, occur with both gestational age and parity. Moreover, parity (14) and age (15) may themselves affect the sex ratio at birth. However, controlling for several critical factors did not alter the estimates indicating a robustness of the eating disorder subtype association with sex ratio at birth. Despite the large sample, the small numbers of participants reporting anorexia nervosa and EDNOS-P contribute to large variability in their estimated sex ratios at birth. Replication of analyses using birth registries and retrospective histories are required to corroborate the observed association.

Nonetheless, these data suggest that maternal eating disorders may influence offspring sex and that the direction of effect may differ depending on eating disorder subtype. Whether this effect stems from maternal diet, stress, hormonal levels or other factors affiliated with both eating disorders and sex ratio remains unknown. Furthermore, the sex ratio differences across subtypes can exist at the primary sex ratio level. It is also unknown whether the effects of a maternal eating disorder on sex ratio operate periconceptually, for example by preferential fertilization of the oocyte by $\mathrm{x}$ - or $\mathrm{y}$ - bearing sperm $(5,16)$, or post-conceptionally, in anorexia and bulimia nervosa, with an increased loss of male fetuses because of an increased susceptibility to poor nutrition or intrauterine stressors (17). If confirmed, this finding could provide evidence in formulating other hypotheses regarding the consequences of eating disorders and possible determinants of sex ratio at birth.

\section{Acknowledgements}

This research was supported by the National Institutes of Health Grants (HD047186) to CMB and the MoBa study is supported by the Norwegian Ministry of Health, NIH/NIEHS (grant no. N01 - ES - 85433), NIH/NINDS (grant no. 1 UO1 NS 047537-01) and Norwegian Research Council/FUGE (grant no. 151918/S10). The donations of questionnaire data and biological material from MoBa participants is gratefully acknowledged.

\section{References}

1. Cagnacci A, Renzi A, Arangino S, Alessandrini C, Volpe A. Influences of maternal weight on the secondary sex ratio of human offspring. Hum Reprod 2004;19:442-4. [PubMed: 14747194]

2. Catalano R, Bruckner T, Hartig T, Ong M. Population stress and the Swedish sex ratio. Paediatr Perinat Epidemiol 2005;19:413-20. [PubMed: 16269068]

3. Obel C, Henriksen TB, Secher NJ, Eskenazi B, Hedegaard M. Psychological distress during early gestation and offspring sex ratio. Hum Reprod 2007;22:3009-12. [PubMed: 17768170]

4. Mathews F, Johnson P, Neil A. You are what your mother eats: evidence for maternal preconception diet influencing foetal sex in humans. Proc Biol Sci 2008;275:1661-1668. [PubMed: 18430648]

5. Rosenfeld C, Roberts R. Maternal diet and other factors affecting offspring sex ratio: A review. Biol Reprod 2004;71:1063-70. [PubMed: 15229140]

6. Bulik CM, Von Holle A, Hamer R, Knoph Berg C, Torgersen L, Magnus P, et al. Patterns of remission, continuation and incidence of broadly defined eating disorders during early pregnancy in the Norwegian Mother and Child Cohort Study (MoBa). Psychol Med 2007;37:1109-18. [PubMed: 17493296]

7. Magnus P, Irgens L, Haug K, Nystad W, Skjaerven R, Stoltenberg C. Cohort profile: The Norwegian Mother and Child Cohort Study (MoBa). Int J Epidemiol 2006;35:1146-50. [PubMed: 16926217] 
8. Irgens L, Bergsjø P, Lie R. The Medical Birth Registry of Norway. Epidemiological research and surveillance throughout 30 years. Acta Obstet Gynecol Scand 2000;79:435-9. [PubMed: 10857866]

9. Reichborn-Kjennerud T, Bulik C, Tambs K, Harris J. Genetic and environmental influences on binge eating in the absence of compensatory behaviors: a population-based twin study. Int J Eat Disord 2004;36:307-14. [PubMed: 15478129]

10. American Psychiatric Association. Diagnostic and Statistical Manual for Psychiatric Disorders. Vol. Fourth. Washington, DC: American Psychiatric Press; 1994.

11. SAS Institute Inc. SAS/STAT® Software: Version 9. Cary, NC: SAS Institute, Inc; 2004.

12. Parazzini F, La Vecchia C, Levi F, Franceschi S. Trends in male:female ratio among newborn infants in 29 countries from five continents. Hum Reprod 1998;13:1394-6. [PubMed: 9647579]

13. Vatten L, Skjaerven R. Offspring sex and pregnancy outcome by length of gestation. Early Hum Devel 2004;76:47-54. [PubMed: 14729162]

14. Beratis N, Asimacopoulou A, Varvarigou A. Association of secondary sex ratio with smoking and parity. Fertil Steril 2008;89:662-7. [PubMed: 17517408]

15. Orvos H, Kozinszky Z, Bártfai G. Natural variation in the human sex ratio. Hum Reprod 2001;16:803. [PubMed: 11278239]

16. Jongbloet P. Over-ripeness ovupathy. A challenging hypothesis for sex ratio modulation. Human Reprod 2004;19:269-774.

17. Crawford M, Doyle W, Meadows N. Gender differences at birth and differences in fetal growth. Hum Reprod 1987;2:517-20. [PubMed: 3667908] 
Bulik et al.

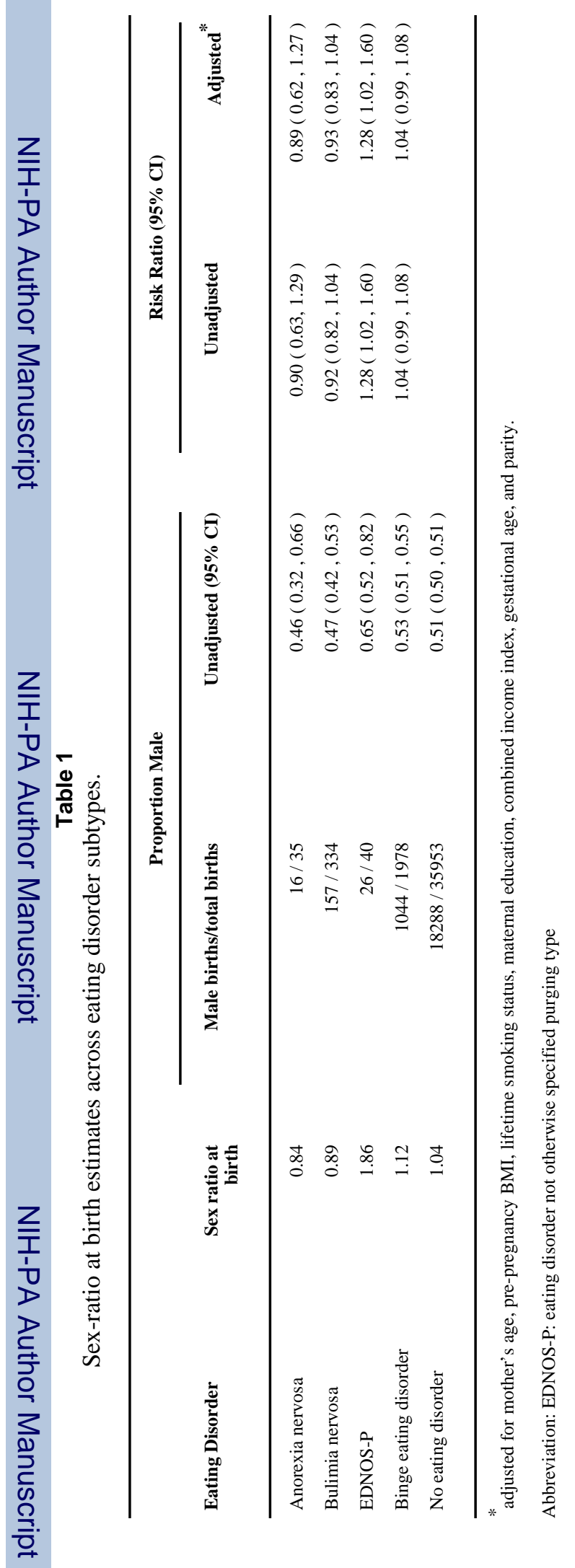

